\title{
PURE-LAMP Procedure for the Diagnosis of Extrapulmonary Tuberculosis: A Case Series
}

\author{
Yuji Kouzaki ${ }^{1}$, Kei Mikita ${ }^{1}$, Takuya Maeda ${ }^{1}$, Masashi Ishihara ${ }^{1}$, Shuichi Kawano ${ }^{1}$, \\ Yuji Fujikura ${ }^{1}$, Kazuo Imai ${ }^{1}$, Soichiro Kanoh ${ }^{1}$, Kazuho Utsunomiya ${ }^{2}$, \\ Masahiro Inoue $^{3}$, Yasushi Miyahira ${ }^{4}$ and Akihiko Kawana ${ }^{1}$
}

\begin{abstract}
Although the polymerase chain reaction is effective for the diagnosis of extrapulmonary tuberculosis (EPTB), it is typically unavailable in resource-limited situations. In contrast, the loop-mediated isothermal amplification (LAMP) assay is a relatively cost-effective and accessible method. Additionally, when combined with the procedure for ultra-rapid extraction (PURE) kit, which enables simple DNA extraction, LAMP can detect Mycobacterium tuberculosis in sputum within 1.5 hours using a simple procedure. In this study, we investigated the utility of the PURE-LAMP technique to diagnose three cases of EPTB and showed that it may potentially be a valuable tool for the diagnosis of EPTB.
\end{abstract}

Key words: loop-mediated isothermal amplification, Mycobacterium tuberculosis, extrapulmonary tuberculosis, procedure for ultra-rapid extraction, diagnosis

(Intern Med 54: 1447-1450, 2015)

(DOI: 10.2169/internalmedicine.54.4615)

\section{Introduction}

Extrapulmonary tuberculosis (EPTB) accounts for approximately $25 \%$ of all cases of tuberculosis (TB) worldwide and it occurs in an even higher proportion of cases of human immunodeficiency virus (HIV) infection $(1,2)$. Microscopy and culturing constitute the current gold standards for diagnosing $\mathrm{TB}$, but the rate of positive results varies considerably for suspected EPTB samples, from 1 to $37 \%$ for smear microscopy and 12 to $80 \%$ for culturing, depending on the type of body fluid examined (3). Therefore, these methods seem to lack sensitivity in the diagnosis of EPTB. Additionally, culturing remains a time-consuming process.

Given the global increase in the number of multidrugresistant TB cases, the early and accurate detection of Mycobacterium tuberculosis (MTb) is of increasing importance for management and timely treatment. Currently, tests involving nucleic acid amplification (NAA) such as poly- merase chain reaction (PCR) offer high performance, rapid results for detecting $M T b$, including in the diagnosis of EPTB $(4,5)$ and are increasingly being regarded as the global standard detection procedure. However, these sophisticated diagnostic tools require not only experience and expertise to run, but also expensive laboratory equipment. As such, they tend to be unavailable in settings with limited clinical resources.

Loop-mediated isothermal amplification (LAMP) may be a practical alternative to other NAA techniques for $M T b$ detection because it does not require any expensive devices or detection instruments. LAMP was first reported in 2000 (6) and apart from its specificity, simplicity and rapidity of use, the LAMP reaction is potentially a very useful diagnostic procedure because it does not require strict purification of DNA samples, which are used as a template. To make LAMP detection of the $M T b$ complex simple for diagnostic purposes, the procedure for ultra-rapid extraction (PURE) kit, which is commercially available and specifically de-

\footnotetext{
${ }^{1}$ Division of Infectious Diseases and Pulmonary Medicine, Department of Internal Medicine, National Defense Medical College, Japan, ${ }^{2}$ Department of Otolaryngology-Head and Neck Surgery, National Defense Medical College, Japan, ${ }^{3}$ Department of Orthopaedic Surgery, National Defense Medical College, Japan and ${ }^{4}$ Department of Global Infectious Diseases and Tropical Medicine, National Defense Medical College, Japan Received for publication November 28, 2014; Accepted for publication January 8, 2015

Correspondence to Dr. Takuya Maeda, tmaeda@ndmc.ac.jp
} 

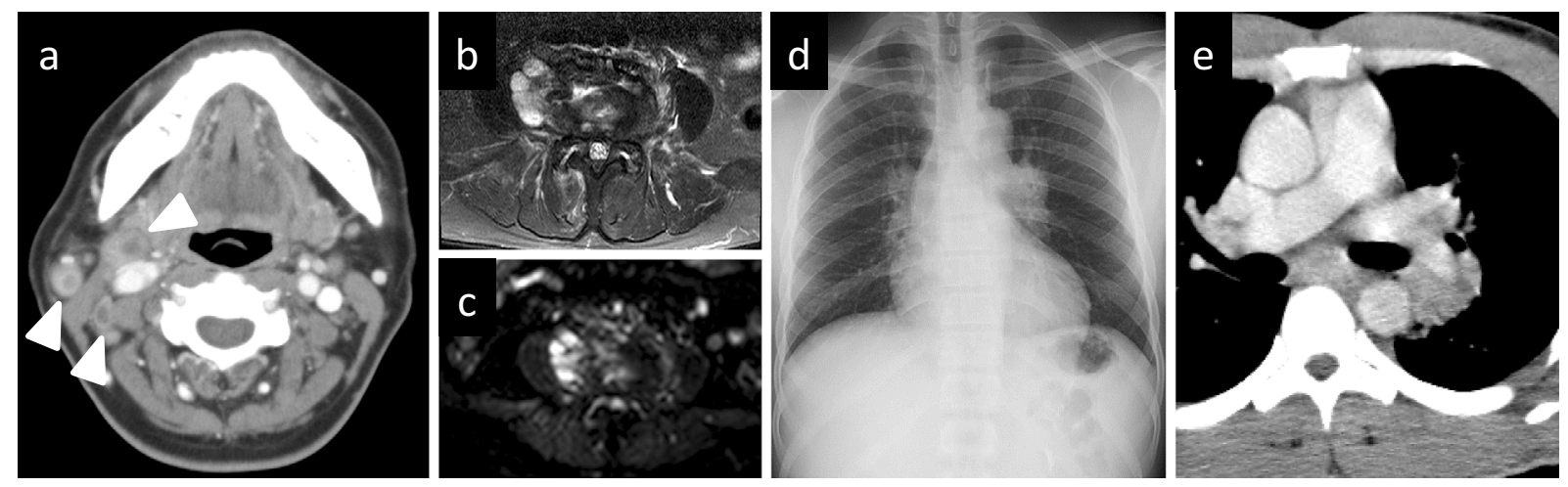

Figure 1. Imaging findings in the three cases. (a) Case 1: contrast-enhanced computed tomography (CT) scan shows multiple enlarged lymph nodes with central non-enhanced regions (arrows). (b) Case 2: axial T2-weighted image shows areas of hyperintensity in the $L 4 / 5$ disc space and $L 5$ vertebral body and (c) diffusion-weighted imaging shows diffusion restriction, presumably indicating abscess accumulation in the disc space extending to within the $\mathrm{L} 5$ vertebral body. (d) Case 3: chest roentgenogram shows unilateral enlargement of the left hilum and (e) contrast-enhanced CT scan shows mediastinal and left hilar lymphadenopathy with heterogeneous enhancement.

Table. Clinical Features and Laboratory Findings for the Diagnosis of Extrapulmonary Tuberculosis in Three Cases.

\begin{tabular}{|c|c|c|c|c|c|c|c|}
\hline Case & $\begin{array}{c}\text { Age } \\
\text { (years) }\end{array}$ & Diagnosis & Sample & $\mathrm{ZN}$ & Culture & PCR & LAMP \\
\hline 1 & 41 & $\begin{array}{l}\text { Tuberculous } \\
\text { lymphadenitis }\end{array}$ & $\begin{array}{l}\text { Swabbing } \\
\text { (Excised lymph node) }\end{array}$ & + & + & + & + \\
\hline 2 & 84 & $\begin{array}{l}\text { Tuberculous } \\
\text { lumbar spondylitis }\end{array}$ & $\begin{array}{l}\text { Aspiration } \\
\text { (Cutaneous drainage) }\end{array}$ & - & + & + & + \\
\hline 3 & 23 & $\begin{array}{l}\text { Tuberculous } \\
\text { lymphadenitis }\end{array}$ & $\begin{array}{l}\text { Aspiration } \\
\text { (EBUS-TBNA) }\end{array}$ & - & + & + & + \\
\hline
\end{tabular}

ZN: Ziehl-Neelsen staining, EBUS-TBNA: endobronchial ultrasound-guided transbronchial needle aspiration

signed for LAMP (Eiken Chemical, Tokyo, Japan), was initially developed to extract DNA from sputum samples and to eliminate inhibitory substances (PURE-TB-LAMP). In a clinical trial of PURE-TB-LAMP in Japan, Mitarai et al. found it to be a simple, effective and rapid test for TB (7). The procedure has also been evaluated in the diagnosis of malaria $(8,9)$, cutaneous leishmaniasis $(10)$ and pneumocystis pneumonia (11). It is expected to become a widely used diagnostic technique requiring only limited equipment and manpower, and to be especially useful in the field setting.

We herein report the application of the PURE-TB-LAMP method using clinical samples other than sputum obtained in suspected EPTB cases and demonstrate the potential of this alternative technique for diagnosing EPTB.

\section{Case Reports}

Three individuals of Filipino, Japanese and Ghanaian origin who were diagnosed with EPTB were included in this study. None had been diagnosed with HIV infection or had a history of pulmonary tuberculosis. The result of an interferon- $\gamma$ release assay against TB was positive in all patients (12); therefore, all were suspected to have EPTB at the time of admission to our hospital.

\section{Case 1}

A 41-year-old Filipina presented with enlarged painful lymph nodes on the right side of her neck. A biopsy was taken to confirm the diagnosis of tuberculous lymphadenitis (Fig. 1a). The specimen was an enlarged lymph node measuring $21 \times 18 \times 13 \mathrm{~mm}$ in size. A histopathological examination confirmed tuberculous lymphadenitis. The specimens collected by swabbing the cut surface of the extracted lymph node were positive for acid-fast bacilli under microscopy with Ziehl-Neelsen (ZN) staining and showed positive results on mycobacterium culture examination with the BacT/ ALERT $^{\circledR}$ three-dimensional microbial detection system (bioMerieux, Nurtingen, Germany). M. tuberculosis DNA was unequivocally identified in the positive culture and clinical specimens by PCR using the Cobas TaqMan MTB test kit (Roche Diagnostics GmbH, Mannheim, Germany) (Table) (13). Samples for the LAMP procedure were obtained by wiping the cut surface of the extracted lymph node with a sterile cotton swab.

\section{Case 2}

An 81-year-old Japanese man was referred to our hospital with an approximately 2-month history of lower back pain. 

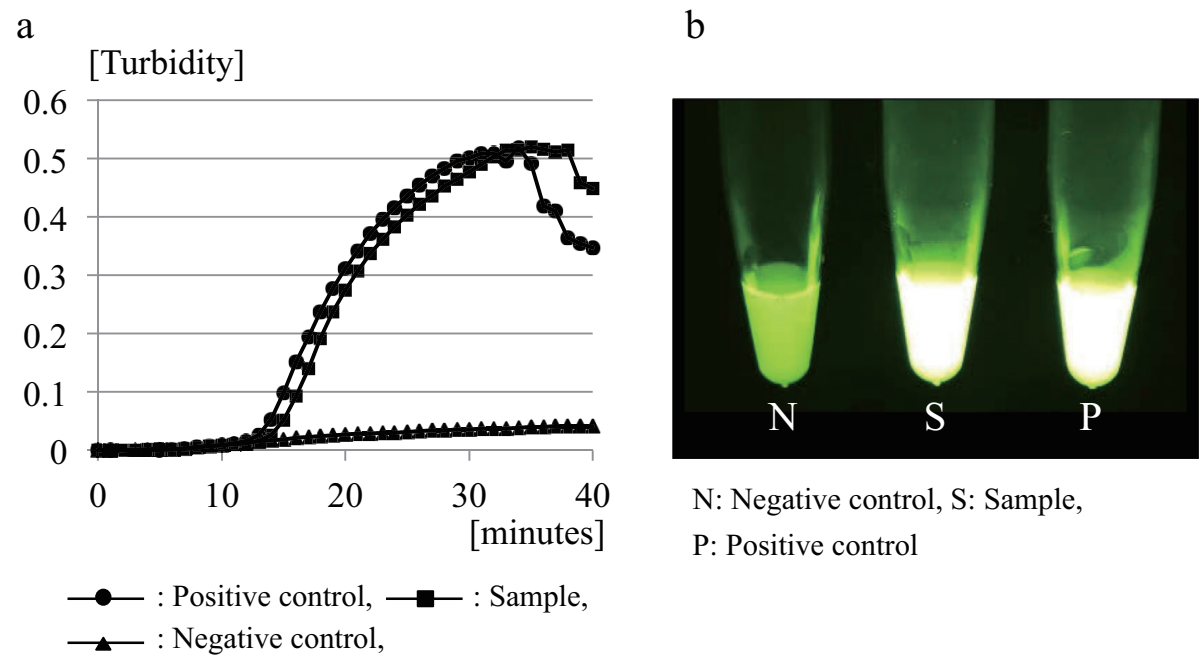

N: Negative control, S: Sample,

P: Positive control

Figure 2. The loop-mediated isothermal amplification (LAMP) assay. (a) Time-related changes in turbidity over the course of LAMP. (b) Visual detection of the LAMP product under UV light. The sample and positive control were both positive.

Magnetic resonance imaging revealed areas of T2 hyperintensity in the L4/5 disc space and L5 vertebral body (Fig. 1b). Diffusion-weighted imaging showed a diffusion restriction in the affected area, presumably indicating abscess accumulation in the disc space extending to within the L5 vertebral body (Fig. 1c). Percutaneous drainage was performed to obtain specimens to confirm the diagnosis of tuberculous lumbar spondylitis. The specimens were examined by microscopy and microbiological culturing. Although the smears were negative for acid-fast bacilli, culturing for mycobacterium using the BacT/ALERT ${ }^{\circledR}$ system was positive. M. tuberculosis DNA was unequivocally identified in the clinical specimens by PCR using the Cobas TaqMan MTB test kit (Table). A drop of the abscess fluid was used as the sample for the PURE-TB-LAMP procedure.

\section{Case 3}

A 23-year-old Ghanaian man who developed mediastinal lymphadenopathy was referred our hospital. A chest roentgenogram showed unilateral enlargement of the left hilum (Fig. 1d), and a contrast-enhanced computed tomography scan showed mediastinal and left hilar lymphadenopathy with heterogeneous enhancement (Fig. 1e). We performed bronchoscopy including endobronchial ultrasound-guided transbronchial needle aspiration (EBUS-TBNA) to obtain a purulent specimen from a left hilar lymph node to confirm the diagnosis of tuberculous lymphadenitis. The smears with $\mathrm{ZN}$ staining were negative for acid-fast bacilli while the culturing for mycobacterium using the BacT/ALERT ${ }^{\circledR}$ system was positive. M. tuberculosis DNA was unequivocally identified in the positive culture and clinical specimens by PCR using the Cobas TaqMan MTB test kit (Table). A sample from the purulent specimen obtained by EBUS-TBNA was used for the PURE-LAMP procedure.

\section{Purification and amplification of $M$. tuberculosis DNA with PURE-LAMP}

We used the PURE kit to isolate target DNA for use in LAMP. The samples derived from the three patients were suspended in or added to $960 \mu \mathrm{L}$ of DNA extraction solution and incubated on a heat-block at $100^{\circ} \mathrm{C}$ for 5 minutes. DNA was rapidly extracted using the PURE kit in $30 \mu \mathrm{L}$ of solution (7). Amplification of the specific MTb-DNA with a commercially available LAMP kit (Eiken Chemical, Tokyo, Japan) was performed at $67^{\circ} \mathrm{C}$ for 40 minutes according to the manufacturer's instructions. Positive results were detected by real-time measurement of turbidity with the LA200 turbidimeter (Eiken Chemical) (Fig. 2a) in cases 2 and 3 and by the naked eye (Fig. 2b) in all cases. Identification of specific MTb-DNA by the PURE-TB-LAMP procedure consistently took only 90 minutes to complete. This study was approved by the National Defense Medical College Research Ethics Committee (Approval No. 2098).

\section{Discussion}

The differential diagnosis of EPTB is extensive, including malignancy and various infections, which makes distinguishing EPTB from other etiologies based on the clinical background alone difficult. The recommended initial procedures for confirming a diagnosis of EPTB are imaging and fine needle biopsy with smear microscopy and culturing; however, the overall sensitivity of these methods is low because of the paucibacillary nature of EPTB and the uneven distribution of the pathogen in the lesions. Other procedures available for confirming the diagnosis of EPTB include fine needle aspiration (FNA) or excisional biopsies for examination by smear and ZN stain, culture, molecular analysis and pathology. The sensitivity of these confirmatory procedures varies considerably, however, ranging from 0 to $77.8 \%$ with 
ZN stain, 8 to $80 \%$ with tissue culture and 33 to $94.6 \%$ with PCR of the biopsied tissue $(14,15)$. It has been suggested that a combination of FNA and PCR might increase the sensitivity of the TB lymphadenitis diagnosis (16), but this would still be time-consuming and would require specialized equipment for confirmation of the EPTB diagnosis.

A recent meta-analysis of the diagnostic accuracy of LAMP for pulmonary TB revealed a sensitivity of $80.0 \%$ [95\% confidence interval (CI), 78-83\%] and a specificity of 96.0\% (95.0-97.0\%) (17). Mitarai et al. reported the sensitivity of PURE-TB-LAMP in smear- and culture-positive sputum specimens to be $98.2 \%$ and that in smear-negative but culture-positive sputum specimens to be $55.6 \%$ (7). Ou et al. demonstrated that the sensitivity of the PURE-TBLAMP method based on spot sputum in smear-negative and culture-positive patients was $53.81 \%$, the overall sensitivity was $70.67 \%$ and the specificity was $98.32 \%$ (18); these results are similar to those from the PCR protocol (19). Unfortunately, LAMP is known to yield false-negative results, although in this study we were able to detect TB-DNA with PURE-LAMP in two smear-negative samples. Additionally, PURE-TB-LAMP can be performed quickly, within 90 minutes including the time required for DNA preparation, and easily with only a heat-block required throughout the protocol. In this regard, even if the sensitivity of the test is not very high, it might be worthwhile performing it to avoid further invasive procedures. At this time, we would recommend that the diagnosis provided by PURE-TB-LAMP be verified by another diagnostic procedure such as imaging.

The PURE device consists of interlocking plastic components and provides a closed system for rapid DNA purification from clinical samples without using any other laboratory equipment (e.g., micropipette or centrifuge). Compared with other NAA techniques such as PCR, the LAMP assay is more resistant to contamination with reaction-inhibitory substances often present in clinical samples. Since a large amount of contaminating substances inhibits the LAMP reaction, we previously evaluated the ability of PURE to eliminate the substrates that inhibit the LAMP reaction, including whole blood and debris from swab samples of a skin ulcer (10). We found that the PURE kit could be used to extract DNA from these clinical sample types and adequately eliminate the inhibitory substances, making it easy to detect DNA by combining it with the LAMP reaction.

Although the clinical effectiveness of the PURE method combined with LAMP must still be further proven, we have demonstrated the potential of this method for diagnosing EPTB as well as pulmonary TB. Given the advantages of PURE-TB-LAMP (e.g., the entire diagnostic procedure can be performed within 90 minutes and no sophisticated laboratory equipment is needed), we anticipate that it will be suitable not only for use in resource-limited situations, but also in well-equipped health facilities.
The authors state that they have no Conflict of Interest (COI).

\section{References}

1. Jones BE, Young SM, Antoniskis D, Davidson PT, Kramer F, Barnes PF. Relationship of the manifestations of tuberculosis to CD4 cell counts in patients with human immunodeficiency virus infection. Am Rev Respir Dis 148: 1292-1297, 1993.

2. Nelson LJ, Wells CD. Global epidemiology of childhood tuberculosis. Int J Tuberc Lung Dis 8: 636-647, 2004.

3. Sharma SK, Mohan A. Extrapulmonary tuberculosis. Indian J Med Res 120: 316-353, 2004.

4. Boehme CC, Nicol MP, Nabeta P, et al. Feasibility, diagnostic accuracy, and effectiveness of decentralised use of the Xpert MTB/ RIF test for diagnosis of tuberculosis and multidrug resistance: a multicentre implementation study. Lancet 377: 1495-1505, 2011.

5. Tortoli E, Russo C, Piersimoni C, et al. Clinical validation of Xpert MTB/RIF for the diagnosis of extrapulmonary tuberculosis. Eur Respir J 40: 442-447, 2012.

6. Notomi T, Okayama H, Masubuchi H, et al. Loop-mediated isothermal amplification of DNA. Nucleic Acids Res 28: E63, 2000.

7. Mitarai S, Okumura M, Toyota E, et al. Evaluation of a simple loop-mediated isothermal amplification test kit for the diagnosis of tuberculosis. Int J Tuberc Lung Dis 15: 1211-1217, 2011.

8. Polley SD, Gonzalez IJ, Mohamed D, et al. Clinical evaluation of a LAMP test kit for diagnosis of imported malaria. J Infect Dis 208: 637-644, 2013.

9. Hopkins H, González IJ, Polley SD, et al. Highly sensitive detection of malaria parasitemia in an endemic setting: performance of a new LAMP kit in a remote clinic in Uganda. J Infect Dis 208: 645-652, 2013.

10. Mikita K, Maeda T, Yoshikawa S, Ono T, Miyahira Y, Kawana A. The Direct Boil-LAMP method: a simple and rapid diagnostic method for cutaneous leishmaniasis. Parasitol Int 63: 785-789, 2014.

11. Kawano S, Maeda T, Suzuki T, et al. Loop-mediated isothermal amplification with the Procedure for Ultra Rapid Extraction kit for the diagnosis of pneumocystis pneumonia. J Infect Chemother 21: 224-226, 2015.

12. Meier T, Eulenbruch HP, Wrighton-Smith P, Enders G, Regnath T. Sensitivity of a new commercial enzyme-linked immunospot assay (T SPOT-TB) for diagnosis of tuberculosis in clinical practice. Eur J Clin Microbiol Infect Dis 24: 529-536, 2005.

13. Kim JH, Kim YJ, Ki CS, Kim JY, Lee NY. Evaluation of Cobas TaqMan MTB PCR for detection of Mycobacterium tuberculosis. J Clin Microbiol 49: 173-176, 2011.

14. Mittal P, Handa U, Mohan H, Gupta V. Comparative evaluation of fine needle aspiration cytology, culture, and PCR in diagnosis of tuberculous lymphadenitis. Diagn Cytopathol 39: 822-826, 2011.

15. Derese Y, Hailu E, Assefa T, et al. Comparison of PCR with standard culture of fine needle aspiration samples in the diagnosis of tuberculosis lymphadenitis. J Infect Dev Ctries 6: 53-57, 2012.

16. Singh KK, Muralidhar M, Kumar A, et al. Comparison of in house polymerase chain reaction with conventional techniques for the detection of Mycobacterium tuberculosis DNA in granulomatous lymphadenopathy. J Clin Pathol 53: 355-361, 2000.

17. Yuan LY, Li Y, Wang M, Ke ZQ, Xu WZ. Rapid and effective diagnosis of pulmonary tuberculosis with novel and sensitive loopmediated isothermal amplification (LAMP) assay in clinical samples: a meta-analysis. J Infect Chemother 20: 86-92, 2014.

18. Ou X, Li Q, Xia H, et al. Diagnostic accuracy of the PURELAMP test for pulmonary tuberculosis at the county-level laboratory in China. PLoS One 9: e94544, 2014.

19. Suffys P, Palomino JC, Cardoso Leão S, et al. Evaluation of the polymerase chain reaction for the detection of Mycobacterium tuberculosis. Int J Tuberc Lung Dis 4: 179-183, 2000.

(C) 2015 The Japanese Society of Internal Medicine http://www.naika.or.jp/imonline/index.html 